Philip D. Loewen, Department of Mathematics, University of British Columbia, Vancouver, B.C., Canada, V6T 1 Z2 (email: loew@math.ubc.ca)

Xianfu Wang`, Department of Mathematics, University of British Columbia, Vancouver, B.C., Canada, V6T 1 Z2 (email: xwang@math.ubc.ca)

\title{
TYPICAL PROPERTIES OF LIPSCHITZ FUNCTIONS
}

\begin{abstract}
In terms of Baire category, a typical real-valued Lipschitz function on a finite dimensional space has a local minimum at every point of a dense subset of the domain, and a Dini subdifferential that is either singleton or empty at all points. Moreover, its Dini subdifferential is empty outside a set of first category. Hence a typical Lipschitz function has no points of subdifferential regularity.
\end{abstract}

\section{Introduction}

In [8], Sciffer constructs a real-valued Lipschitz function on the line that is nowhere Clarke regular. Here we describe a natural setting in which this behavior can be regarded as typical, even for a function of several variables. In fact, we show that for a typical Lipschitz function,

- the Dini subdifferential never contains more than one element,

- the Dini subdifferential is empty outside a set of first category,

- local minimizers are dense in the domain, and

- the Clarke subdifferential is maximal at every point.

Key Words: Lipschitz function, Dini subdifferential, Baire category, dense minimizers, nonangularity, subdifferential regularity

Mathematical Reviews subject classification: $49 \mathrm{~J} 52$

Received by the editors August 23, 2000

* Research supported by NSERC

${ }^{\dagger}$ Research supported by NSERC post-doctoral fellowship 
Throughout this paper we deal with a fixed finite-dimensional normed space $X$ with closed unit ball $\mathbb{B}$, topological dual $X^{*}$, and closed dual ball $\mathbb{B}^{*}$, and with a fixed compact convex set $C \subset X^{*}$ such that int $C \neq \emptyset$. We write

$$
\operatorname{Lip}_{C}:=\left\{f: X \rightarrow R: f(x)-f(y) \leq \sigma_{C}(x-y) \text { for all } x, y \in X\right\},
$$

where $\sigma_{C}(x):=\max \{\langle c, x\rangle: c \in C\}$ is the support function of $C$. The boundedness of $C$ implies that every function in $\operatorname{Lip}_{C}$ is Lipschitzian in the usual sense. Indeed, when $C=K \mathbb{B}^{*}$ for some $K>0, \operatorname{Lip}_{C}$ is the usual set of $K$-Lipschitz functions on $X$. We define a complete metric space $\left(\operatorname{Lip}_{C}, \rho\right)$ by setting $\rho_{n}(f, g):=\sup \{|f(x)-g(x)|: x \in n \mathbb{B}\}$ and

$$
\rho(f, g):=\sum_{n=1}^{\infty} \frac{1}{2^{n}} \min \left\{\rho_{n}(f, g), 1\right\}, \quad f, g \in \operatorname{Lip}_{C} .
$$

Note that a sequence of functions $\left\langle f_{k}\right\rangle$ in $\operatorname{Lip}_{C}$ converges to $f$ in the metric $\rho$ if and only if $f_{k}$ converges to $f$ uniformly on each compact subset of $X$. We call a property typical in $\operatorname{Lip}_{C}$ when it holds throughout some subset of $\operatorname{Lip}_{C}$ whose complement has first category.

Subgradients. For $f \in \operatorname{Lip}_{C}$ and $x \in X$, the lower Dini directional derivative and Dini subgradient are defined by

$$
\begin{aligned}
f_{+}(x ; v) & :=\liminf _{t \rightarrow 0^{+}} \frac{f(x+t v)-f(x)}{t}, \\
\widehat{\partial} f(x) & :=\left\{x^{*} \in X^{*}: f_{+}(x ; v) \geq\left\langle x^{*}, v\right\rangle \forall v \in X\right\} .
\end{aligned}
$$

(Other authors call $\widehat{\partial} f$ the Fréchet subdifferential or the regular subdifferential.) The set $\widehat{\partial} f(x)$ is closed, but may well be empty. Thus we must contend with the set $\operatorname{dom}(\widehat{\partial} f):=\{x \in X: \widehat{\partial} f(x) \neq \emptyset\}$. We will also refer to the upper Dini directional derivative $f^{+}=-(-f)_{+}$, given explicitly by

$$
f^{+}(x ; v):=\limsup _{t \rightarrow 0^{+}} \frac{f(x+t v)-f(x)}{t} .
$$

The general subgradient of $f$ at $x$ is, by definition,

$$
\partial f(x):=\bigcap_{\delta>0} \operatorname{cl}\left\{z^{*}: z^{*} \in \widehat{\partial} f(z),\|z-x\|<\delta\right\} .
$$


The Clarke directional derivative and subgradient of $f$ at $x$ are

$$
\begin{aligned}
f^{\circ}(x ; v) & :=\limsup _{y \rightarrow x, t \downarrow 0} \frac{f(y+t v)-f(y)}{t}, v \in X, \\
\partial^{\circ} f(x) & :=\left\{x^{*} \in X^{*}:\left\langle x^{*}, v\right\rangle \leq f^{\circ}(x ; v) \forall v \in X\right\} .
\end{aligned}
$$

Since $f \in \operatorname{Lip}_{C}$, we have $\partial^{\circ} f \equiv \operatorname{co} \partial f$. (See $[3,6]$ for details and discussion.) Recall that $f \in \operatorname{Lip}_{C}$ is called subdifferentially regular [or Clarke regular] at $x$ when $\widehat{\partial} f(x)=\partial^{\circ} f(x)$.

Our purpose in this note is to show that while a typical function in $\operatorname{Lip}_{C}$ has a local minimum on a dense subset of points in its domain, making its Clarke subdifferential large everywhere, its Dini subdifferential is empty outside a set of first category, and never contains more than one element. Taken together, these statements imply that a typical Lipschitz function is nowhere subdifferentially regular.

\section{Density of Local Minimizers}

Given a continuous function $\phi: X \rightarrow \mathbb{R}$, let $\Sigma(\phi)$ denote the set of points in $X$ where $\phi$ has a local minimum.

Theorem 1. For each $c \in \operatorname{int}(C)$, the following set is residual in $\left(\operatorname{Lip}_{C}, \rho\right)$.

$$
G(c):=\left\{f \in \operatorname{Lip}_{C}: \Sigma(f-c) \text { is dense in } X\right\} .
$$

$($ Here $(f-c)(y)=f(y)-\langle c, y\rangle$.)

Proof. Fix $c \in \operatorname{int} C$. For each fixed $x \in X$, define a sequence of sets indexed by $n \in \mathbb{N}$.

$$
G_{x}^{n}:=\left\{f \in \operatorname{Lip}_{C}: \Sigma(f-c) \cap \operatorname{int}\left(x+\frac{1}{n} \mathbb{B}\right) \neq \emptyset\right\} .
$$

We claim that each set $\operatorname{int}\left(G_{x}^{n}\right)$ is dense in $\operatorname{Lip}_{C}$. To prove this, choose an arbitrary $f \in \operatorname{Lip}_{C}$ and $\varepsilon \in(0,1)$. Define $h(y):=f(x)-\varepsilon+\sigma_{C}(y-x)$ for $y \in X$, and let $h_{1}:=\min \{f, h\}$. Clearly $h$ and $h_{1}$ lie in $\operatorname{Lip}_{C}$. Since $f(y)-f(x) \leq \sigma_{C}(y-x)$ for every $y \in X$, we have

$$
f(y)-\varepsilon \leq f(x)-\varepsilon+\sigma_{C}(y-x)=h(y) ;
$$

so $f \geq h_{1} \geq f-\varepsilon$ and $\rho\left(h_{1}, f\right) \leq \varepsilon$.

As $h(x)<f(x)$, by continuity there is some $\delta \in(0,1 / n)$ sufficiently small that $h(y)<f(y)$ for all $y$ in $x+\delta \mathbb{B}$, whence $h_{1}(y)=h(y)$ for all $y \in x+\delta \mathbb{B}$. 
This implies that $h_{1}-c$ has a local minimum at $x$, since every $y \in x+\delta \mathbb{B}$ obeys

$$
h_{1}(y)-\langle c, y\rangle=h_{1}(x)-\langle c, x\rangle+\left[\sigma_{C}(y-x)-\langle c, y-x\rangle\right]
$$

and the inequality $\sigma_{C}(y-x) \geq\langle c, y-x\rangle$ holds because $c \in C$. Thus $h_{1} \in G_{x}^{n}$.

Let us show that in fact $h_{1} \in \operatorname{int} G_{x}^{n}$. By hypothesis, there is some $r>0$ such that $c+r \mathbb{B}^{*} \subset C$. Thus (1) implies

$$
m:=\inf \left\{h_{1}(y)-\langle c, y\rangle:\|y-x\|=\delta\right\} \geq h_{1}(x)-\langle c, x\rangle+r \delta .
$$

Let $\alpha:=m-\left(h_{1}(x)-\langle c, x\rangle\right)>0$ and choose $0<\beta<\min \{\alpha / 2,1\}$. Also choose an integer $N>\|x\|+1$, and note that every $g \in \operatorname{Lip}_{C}$ with $\rho\left(g, h_{1}\right)<\beta / 2^{N}$ satisfies $\rho_{N}\left(g, h_{1}\right)<\beta$. When $\|y-x\|=\delta$ we have

$$
\begin{aligned}
& g(y)-\langle c, y\rangle=g(y)-h_{1}(y)+h_{1}(y)-\langle c, y\rangle \geq-\beta+m, \text { and } \\
& g(x)-\langle c, x\rangle=g(x)-h_{1}(x)+h_{1}(x)-\langle c, x\rangle \leq \beta+h_{1}(x)-\langle c, x\rangle .
\end{aligned}
$$

Thus

$$
\begin{aligned}
\inf \{g(y)-\langle c, y\rangle:\|y-x\|=\delta\} & \geq-\beta+m \\
>\beta+h_{1}(x)-\langle c, x\rangle & \geq g(x)-\langle c, x\rangle .
\end{aligned}
$$

Now the continuous function $g-c$ must attain its minimum over the compact set $x+\delta \mathbb{B}$, and the strict inequality above implies that the minimizing point cannot lie on the boundary. Hence this point must actually provide an unrestricted local minimum for $g-c$. Since $\delta<1 / n$, this shows that $g \in G_{x}^{n}$. The same conclusion holds for every $g$ satisfying $\rho\left(g, h_{1}\right)<\beta / 2^{N}$; so indeed $h_{1} \in \operatorname{int} G_{x}^{n}$.

Since $\operatorname{int}\left(G_{x}^{n}\right)$ is open and dense in $\operatorname{Lip}_{C}$, the set $G_{x}:=\bigcap_{n=1}^{\infty} \operatorname{int}\left(G_{x}^{n}\right)$ is dense in $\operatorname{Lip}_{C}$. If $f \in G_{x}$, then for every $n$ there exists $x_{n} \in \operatorname{int}\left(x+n^{-1} \mathbb{B}\right)$ such that $f-c$ attains a local minimum at $x_{n}$. That is, $f-c$ attains a local minimum in every neighborhood of $x$.

Finally, let $Q=\left\{x_{k}: k \in \mathbb{N}\right\}$ be a countable dense subset of $X$. Since each $G_{x_{k}}, k \in \mathbb{N}$, is a dense $G_{\delta}$ by the previous paragraph, the set $G:=\bigcap_{k=1}^{\infty} G_{x_{k}}$ is a dense $G_{\delta}$ in $\operatorname{Lip}_{C}$. Now consider any $f \in G$. Every open set $U$ in $X$ contains some $x_{k}$ in $Q$, and since $f \in G_{x_{k}}$, the function $f-c$ attains a local minimum at some point in $U$. Hence the set of points at which $f-c$ attains a local minimum is dense in $X$. Since $f$ is arbitrary in $G$, the dense $G_{\delta}$ set $G$ defined here is a subset of the set $G(c)$ defined in the theorem statement. Hence $G(c)$ is residual.

Corollary 2 ([1]). The following set is residual in $\left(\operatorname{Lip}_{C}, \rho\right)$.

$$
\left\{f \in \operatorname{Lip}_{C}: \partial^{\circ} f(x)=\partial f(x)=C \forall x \in X\right\} .
$$


Proof. For each $c \in C$, define $G(c)$ as in Theorem 1. If $f \in G(c)$, then $c$ lies in $\widehat{\partial} f(x)$ at every point where $f-c$ has a local minimum, and such points are dense in $X$. Consequently $c \in \partial f(x)$ for every $x \in X$. By taking a countable set $\left\{c_{k}: k \in \mathbb{N}\right\}$ dense in the interior of $C$ we obtain a residual set $G:=\bigcap_{k \in \mathbb{N}} G\left(c_{k}\right)$ such that every $f \in G$ obeys $c_{k} \in \partial f(x)$ for every $k \in \mathbb{N}$ and every $x \in X$. But $\partial f(x)$ is a closed set; so $\partial f(x) \supseteq C$. The reverse inclusion follows immediately from the defining property of $\operatorname{Lip}_{C}$; so equality holds. Hence $\partial f(x)=C=\operatorname{co} C=\operatorname{co} \partial f(x)=\partial^{\circ} f(x)$.

\section{Sparseness of the Dini Subdifferential}

We include a simpler proof of the following lemma due to Giles and Sciffer [4] to make our exposition self-contained. Both the statement and the proof remain valid when $X$ is replaced by an arbitrary separable Banach space.

Lemma 3. For every $f \in \operatorname{Lip}_{C}$, there exists a dense $G_{\delta}$ subset of $X$ in which every point $x$ obeys $f^{\circ}(x ; v)=f^{+}(x ; v) \forall v \in X$.

Proof. For $k \in \mathbb{N}$ and $v \in X$, define

$$
D_{k}^{v}:=\left\{x \in X: \frac{f\left(x+t_{x} v\right)-f(x)}{t_{x}}-f^{\circ}(x ; v)>-\frac{1}{k} \text { for some } t_{x} \in\left(0, \frac{1}{k}\right)\right\} .
$$

Because $f$ is Lipschitz and $-f^{\circ}(\cdot ; v)$ is l.s.c., $D_{k}^{v}$ is open. To show that $D_{k}^{v}$ is dense, let $x \in X$ and $\varepsilon>0$ be given. Choose $y \in X$ such that $\|y-x\|<\varepsilon / 2$ and $f^{\circ}(\cdot ; v)$ is continuous at $y$. (This is possible because $f^{\circ}(\cdot ; v)$ is u.s.c., so $f^{\circ}(\cdot ; v)$ is continuous on a residual subset of $X$ - see [7, Exercise 7.43].) Then choose $\delta \in(0, \varepsilon / 2)$ so small that

$$
f^{\circ}(y ; v)-f^{\circ}(z ; v)>-\frac{1}{2 k} \quad \text { whenever }\|z-y\|<\delta .
$$

The definition of $f^{\circ}(y ; v)$ provides points $z$ and $t_{z}$ satisfying $\|z-y\|<\delta$, $0<t_{z}<1 / k$, and

$$
\frac{f\left(z+t_{z} v\right)-f(z)}{t_{z}}>f^{\circ}(y ; v)-\frac{1}{2 k} .
$$

In conjunction with (2), this implies

$$
\frac{f\left(z+t_{z} v\right)-f(z)}{t_{z}}>f^{\circ}(z ; v)-\frac{1}{k} .
$$


Thus $z \in D_{k}^{v}$; also $\|z-x\|<\varepsilon$; so $D_{k}^{v}$ is dense. It follows that each set $D(v):=\bigcap_{k \in \mathbb{N}} D_{k}^{v}$ is a dense $G_{\delta}$ in $X$. Moreover, for every $x \in D(v)$, each $k \in \mathbb{N}$ has some $0<t_{k}<1 / k$ for which

$$
\frac{f\left(x+t_{k} v\right)-f(x)}{t_{k}}-f^{\circ}(x ; v)>-\frac{1}{k} .
$$

As $k \rightarrow \infty$ here, we obtain $f^{+}(x ; v) \geq f^{\circ}(x ; v)$. But $f^{\circ}(x ; v) \geq f^{+}(x ; v)$ is obvious, so equality holds.

Now take a countable dense subset $\left\{v_{n}: n \in \mathbb{N}\right\}$ of $X$, and consider $D:=$ $\bigcap_{n \in \mathbb{N}} D\left(v_{n}\right)$. This is a dense $G_{\delta}$ in $X$, and for every $x \in D$ we have $f^{+}\left(x ; v_{n}\right)=$ $f^{\circ}\left(x ; v_{n}\right)$ for all $n \in \mathbb{N}$. But both $f^{+}(x, \cdot)$ and $f^{\circ}(x, \cdot)$ are Lipschitz, so in fact $f^{+}(x ; \cdot) \equiv f^{\circ}(x ; \cdot)$, as required.

Theorem 4. The following subset of $\left(\operatorname{Lip}_{C}, \rho\right)$ is residual.

$$
G:=\left\{g \in \operatorname{Lip}_{C}: \operatorname{dom}(\widehat{\partial} g) \text { has first category in } X\right\} .
$$

Proof. We will show $G \supseteq A$, where $A:=\left\{g \in \operatorname{Lip}_{C}: \partial^{\circ} g(x)=C \forall x \in X\right\}$. Recall that $A$ is residual in $\left(\operatorname{Lip}_{C}, \rho\right)$, by Corollary 2. Fix $g \in A$. Then $\partial^{\circ}(-g) \equiv-C$ on $X$, and the set

$$
S:=\left\{x \in X:(-g)^{+}(x ; \cdot) \equiv(-g)^{\circ}(x ; \cdot)\right\}
$$

is residual in $X$ by Lemma 3 . For fixed $x$ in $S$, the definition of the Clarke subgradient gives $(-g)^{+}(x ; v)=(-g)^{\circ}(x ; v)=\sigma_{-C}(v)$; i.e., $g_{+}(x ; v)=-\sigma_{C}(-v)$, for all $v \in X$. Now since $C$ has nonempty interior, the sublinear function $\sigma_{C}$ cannot be dominated by a linear function. Therefore the set

$$
\widehat{\partial} g(x)=\left\{x^{*} \in X^{*}:\left\langle x^{*}, v\right\rangle \leq g_{+}(x ; v)=-\sigma_{C}(-v) \forall v \in X\right\}
$$

must be empty. This shows that $\operatorname{dom}(\widehat{\partial} g)$ is a subset of $X \backslash S$, which implies that $\operatorname{dom}(\widehat{\partial} g)$ has first category in $X$. Hence $g \in G$, as required.

Although Rademacher's theorem asserts that every $g$ in $\operatorname{Lip}_{C}$ is differentiable on a set of full Lebesgue measure, each $g$ in the set $G$ of Theorem 4 is differentiable at most on a set of first category in $X$.

\section{Nonangularity}

A function $f: X \rightarrow R$ is called nonangular at $x$ in direction $v$ when

$$
f_{+}(x ; v) \leq-f_{+}(x ;-v) \text { and }(-f)_{+}(x ; v) \leq-(-f)_{+}(x ;-v) .
$$


We call $f$ nonangular at $x$ when (3) holds for every $v \in X$. This definition extends a concept well known when $X=\mathbb{R}$ : see Bruckner [2].

Theorem 5. The subset of $\operatorname{Lip}_{C}$ consisting of functions nonangular at every point of $X$ is a dense $G_{\delta}$.

Proof. Let us fix $v \in X$ and show that

$$
\begin{aligned}
& A(v):=\left\{f \in \operatorname{Lip}_{C}: f_{+}(x ; v)>-f_{+}(x ;-v) \text { for some } x \in X\right\}, \\
& B(v):=\left\{f \in \operatorname{Lip}_{C}:(-f)_{+}(x ; v)>-(-f)_{+}(x ;-v) \text { for some } x \in X\right\}
\end{aligned}
$$

are $F_{\sigma}$ sets of first category in $\operatorname{Lip}_{C}$. We treat the set $A(v)$, the arguments for $B(v)$ being similar. Since $A(v)=\bigcup_{m=1}^{\infty} A^{m}$, where

$$
A^{m}:=\left\{f \in \operatorname{Lip}_{C}: f_{+}(x ; v)>-f_{+}(x ;-v) \text { for some } x \in m \mathbb{B}\right\},
$$

it suffices to show that each $A^{m}$ is an $F_{\sigma}$ of first category in $\operatorname{Lip}_{C}$. For fixed $p, q \in \mathbb{Q}$ and $n \in \mathbb{N}$, with $p<q$, let

$$
\begin{aligned}
A_{p q n}:=\left\{f \in \operatorname{Lip}_{C}\right. & : \text { some } x \in m \mathbb{B} \text { obeys } \\
& \text { both } \frac{f(x+t v)-f(x)}{t} \leq p \forall t \in\left(-\frac{1}{n}, 0\right) \\
& \text { and } \left.\frac{f(x+t v)-f(x)}{t} \geq q \forall t \in\left(0, \frac{1}{n}\right)\right\} .
\end{aligned}
$$

Clearly $A^{m}=\bigcup_{p, q, n} A_{p q n}$. We check that each $A_{p q n}$ is closed and nowhere dense in $\operatorname{Lip}_{C}$.

To see that $A_{p q n}$ closed, let $\left\{f_{k}\right\}$ be any sequence in $A_{p q n}$ converging in $\operatorname{Lip}_{C}$ to some function $f$. We must show that $f \in A_{p q n}$. For each $k$, there exists $x_{k} \in m \mathbb{B}$ such that

$$
\begin{gathered}
\frac{f_{k}\left(x_{k}+t v\right)-f_{k}\left(x_{k}\right)}{t} \leq p \forall t \in\left(-\frac{1}{n}, 0\right), \\
\frac{f_{k}\left(x_{k}+t v\right)-f_{k}\left(x_{k}\right)}{t} \geq q \forall t \in\left(0, \frac{1}{n}\right) .
\end{gathered}
$$

By passing to a subsequence if necessary (we do not relabel), we may assume that $\left\langle x_{k}\right\rangle$ converges to some point $x \in m \mathbb{B}$. As $k \rightarrow \infty$ along this subsequence, 
(4) and (5) yield

$$
\begin{aligned}
& \frac{f(x+t v)-f(x)}{t} \leq p \forall t \in\left(-\frac{1}{n}, 0\right), \\
& \frac{f(x+t v)-f(x)}{t} \geq q \forall t \in\left(0, \frac{1}{n}\right) .
\end{aligned}
$$

Thus $f \in A_{p q n}$ and $A_{p q n}$ is closed.

To show that $A_{p q n}$ is nowhere dense in $\operatorname{Lip}_{C}$, it suffices to observe that any differentiable function in $\operatorname{Lip}_{C}$ must lie outside $A_{p q n}$ and that differentiable functions are dense in $\operatorname{Lip}_{C}$. Indeed, standard mollification methods show that $C^{\infty}$ functions are dense in $\operatorname{Lip}_{C}$. For example, following [6, page 409], let $\phi: X \rightarrow \mathbb{R}$ be a nonnegative function of class $C^{\infty}$ supported in $\mathbb{B}$ and satisfying $\int_{\mathbb{B}} \phi(x) d x=1$. For given $f \in \operatorname{Lip}_{C}$, define $v_{k}=1 / \int_{X} \phi(k z) d z$ and let

$$
f_{k}(x):=v_{k} \int_{X} f(x-z) \phi(k z) d z=v_{k} \int_{X} f(z) \phi(k(x-z)) d z, k \in \mathbb{N} .
$$

Then $f_{k} \rightarrow f$ uniformly on compact subsets of $X$, and $f_{k} \in C^{\infty}$ for each $k$. Moreover, $f_{k}^{\prime}(x)=v_{k} \int_{X} f^{\prime}(x-z) \phi(k z) d z$. Since $f^{\prime}(x-z) \in C$ almost everywhere (Rademacher's Theorem), while $C$ is compact convex and $v_{k} \int_{X} \phi(k z) d z=1$, we have $f_{k}^{\prime}(x) \in C$ for every $x \in X$. This implies $f_{k} \in \operatorname{Lip}_{C}$.

Now let $\left\{v_{k}: k \in \mathbb{N}\right\}$ be a countable dense subset of $X$, and put

$$
G=\operatorname{Lip}_{C} \backslash \bigcup_{k \in \mathbb{N}}\left[A\left(v_{k}\right) \cup B\left(v_{k}\right)\right] .
$$

This is a dense $G_{\delta}$ in $\operatorname{Lip}_{C}$. For every $f \in G$, we have $f_{+}\left(x ; v_{k}\right) \leq-f_{+}\left(x ;-v_{k}\right)$ and $(-f)_{+}\left(x ; v_{k}\right) \leq-(-f)_{+}\left(x ;-v_{k}\right)$ for all $k \in \mathbb{N}$. But $f_{+}(x ; \cdot)$ and $(-f)_{+}(x ; \cdot)$ are Lipschitzian, so these inequalities extend to every $v$ in $X$. Hence every $f \in G$ is nonangular in every direction, at every point of $X$.

Note that if $f$ is nonangular at $x$ and $x^{*} \in \widehat{\partial} f(x)$, then $\left\langle x^{*}, v\right\rangle=f_{+}(x ; v)$ for all $v$, so $\widehat{\partial} f(x)=\left\{x^{*}\right\}$. Thus the set $\widehat{\partial} f(x)$ contains at most one point, and likewise for $\widehat{\partial}(-f)(x)$. Together with Corollary 2 and Theorem 5 , this observation establishes the following generic complement to the explicit construction of Sciffer [8].

Corollary 6. There is a residual subset $G$ of $\operatorname{Lip}_{C}$ such that every $f \in G$ fails to be subdifferentially regular at every point of $X$, and the same is true for $-f$. 
Remark. When $X=\mathbb{R}$, analogues of Corollary 6 hold in the spaces of bounded continuous functions on $X$ or bounded nondecreasing functions on $X$ using the supremum norm.

Remark. When $X=\mathbb{R}$ and $C=[-1,1]$, Preiss and Tiśer [5] have shown that the set

$$
H:=\left\{f \in \operatorname{Lip}_{C}: \limsup _{y \rightarrow x}\left|\frac{f(y)-f(x)}{y-x}\right|=1 \text { for every } x \in[0,1]\right\}
$$

is residual. Every $f$ in $H$ has $f^{\prime}(x) \in\{-1,1\}$ for almost every $x \in \mathbb{R}$. In conjunction with Corollary 2 and [3, Theorem 2.5.1], this implies that there is a residual subset of $\operatorname{Lip}_{C}$ in which every $f$ is such that both

$$
D_{+}=\left\{x \in \mathbb{R}: f^{\prime}(x)=1\right\} \quad \text { and } \quad D_{-}=\left\{x \in \mathbb{R}: f^{\prime}(x)=-1\right\}
$$

meet every open interval in a set of positive measure.

\section{References}

[1] J. M. Borwein, W. B. Moors, X. Wang, Generalized subdifferentials: a Baire categorical approach, Trans. Amer. Math. Soc., to appear.

[2] A. M. Bruckner, Differentiation of Real Functions, second edition, Providence: American Mathematical Society, 1994.

[3] F. H. Clarke, Optimization and Nonsmooth Analysis, Wiley Interscience, New York, 1983.

[4] J. R. Giles, S. Sciffer, Locally Lipschitz functions are generically pseudoregular on separable Banach spaces, Bull. Austral. Math. Soc. 47 (1993), 205-212.

[5] D. Preiss, J. Tiśer, Points of nondifferentiability of typical Lipschitz functions, Real Anal. Exch. 20 (1994/5), 219-226.

[6] R. T. Rockafellar, R. J-B Wets, Variational Analysis, New York: SpringerVerlag, 1998.

[7] H. L. Royden, Real Analysis, third edition, Englewood Cliffs, N.J.: Prentice-Hall, 1988.

[8] S. Sciffer, Regularity of locally Lipschitz functions on the line, Real Anal. Exch. 20 (1994/1995), 786-798. 
Philip D. Loewen and Xianfu Wang 